\title{
Museos y patrimonio gastronómico
}

\section{Rhina I. Colunge Rosales}

\begin{abstract}
Ahora recoges ajos, acaricia primero ese marfil precioso, huele su fragancia iracunda, entonces deja el ajo picado caer con la cebolla y el tomate hasta que la cebolla tenga color de oro. Mientras tanto se cuecen con el vapor los regios camarones marinos y cuando ya llegaron a su punto, cuando cuajó el sabor en una salsa formada por el jugo del océanoy por el agua clara que desprendió la luz de la cebolla, entonces que entre el congrio y se sumerja en gloria, que en la ollase aceite, se contraiga y se impregne.
\end{abstract}

Pablo Neruda (Fragmento de Oda al caldillo de congrio)

Existen diversos modos de acercarnos al tema del patrimonio gastronómico y de los museos, a sus nexos e interrelaciones. De primera impresión podría parecer a muchos que la gastronomía -al guardar estrecha relación con la alimentación- es materia cercana y común a todos; mientras los museos, sus recintos y colecciones, aparentemente forman parte de las actividades de un grupo más bien privilegiado de personas. Digo aparentemente, porque quizás esta es la visión que aún guardamos de nuestras experiencias en estas instituciones, pero no la función primordial de ellas; sobretodo si recordamos y ponemos en práctica las motivaciones de llevar a cabo una nueva museología. Pero ¿qué hay de nuevo en la nueva museología? como lo recuerda Douret (1999) lo primordial en un "nuevo" museo es el contestar claramente las preguntas del por qué y para quién se conserva, investiga y difunde.

De este modo, no quiero centrar este artículo sólo en aquellos museos que tienen elementos gastronómicos como parte principal de su colección, o los museos de determinados productos alimenticios tradicionales o propios de una región, porque pienso que no sólo ellos tienen vínculos con el patrimonio gastronómico, aunque en su caso este vínculo es más evidente. Muchos aspectos de la vida diaria, entre ellos la gastronomía, guardan una estrecha relación con las colecciones albergadas en los museos o la cualidad ("museality")' detrás de ellos, sean éstas colecciones en torno del arte, historia, arqueología, literatura, etc. Pretendo a lo largo de las siguientes líneas redescubrir estos vínculos, y al hacerlo renovar el acer-

\footnotetext{
1 Al ser la museología una ciencia relativamente nueva, aún no existen acuerdos en cuanto a los equivalentes en otros idiomas de terminología desarrollada hasta el momento en inglés, podriamos hablar quizás de "musealidad" como el equivalente de "museality".
} 
camiento holístico de estos espacios culturales y el debate de su función como actores de cambio social.

\section{I.- Más allá de la conservación de objetos. El museo, el objeto y lo intangible en el objeto.}

\begin{abstract}
"The ultimate materialistic enterprise, museums seem to replace persons with objects, extracting their life and thought, and to presume, by these means, to immortalize the spirit of civilizations past". (Hein, 2000: 51)
\end{abstract}

A pesar de los avances teóricos dentro del campo de la museología los museos siguen siendo considerados por algunos críticos como lugares comparables a áticos o almacenes, en los que los objetos pierden la característica más importante que justamente los hizo ser "musealizados" en un momento dado. Los museos, instituciones creadas en las sociedades occidentales ciertamente centraron durante mucho tiempo su atención en los objetos y en su valoración alrededor de sus características materiales y estructurales. Como lo señala Hilde Hein en la transición biográfica de un objeto entre "antes de" y "después de" su ingreso a un museo, la pieza gana y pierde dimensiones de uso e intercambia valores así como nuevas proporciones de significado. Es por ello que la discusión sobre el valor intangible detrás cada objeto musealizado es de gran importancia. La tendencia, a menos teórica es que este valor a través de una investigación abierta (que cuente de ser posible con la colaboración o participación de sus creadores) y seria, podría acercarse a aquél que el objeto tuvo en su vida primigenia. Si hacemos una reflexión al respecto caeremos en cuenta que lo que en todos los casos se preserva y exhibe es algo inmaterial, intangible:

\begin{abstract}
"Museums are actually warehouses of material things only superficially. At bottom they have always been reservoirs of meaning (...) Museums thus are depots of sorts, though not simply repositories of static relics. They are dynamic transformers in which energy is converted, redistributed, and released" (Hein: 2000: 55).
\end{abstract}

Algunos autores, como por ejemplo Washburn, nos hacen recordar la importancia de esta información intangible detrás de los objetos, como los valores últimos a ser conservados:

"...when we are collecting objects we are collecting information. What I am proposing is that the emphasis be put on the information rather than on the object. The object can become a fetish that if we merely worship it, impedes our understanding of the object itself and its place in our society. And in the process of being worshipped, it sometimes crumbles in our hands." (Washburn, 1984: 6)".

Encontrando un equilibrio entre lo material e inmaterial como valores de nuestro patrimonio cultural a ser igualmente preservados e igualmente importantes para su entendimiento, podríamos utilizar a modo de paralelo el signo lingüístico y los elementos mencionados por Sausurre como parte de éste: significado y significante. En el caso del objeto en los museos, la parte material que percibimos a través de nuestros sentidos sería el significante y la musealidad del objeto su significado; ahora bien, es importante resaltar que este significado, este valor del objeto, dependerá mucho no sólo del contexto en el que éste haya sido producido se presente éste, sino también de la lectura que haga de él el recep-

\footnotetext{
3 -Los museos son en realidad depósitos de objetos materiales sólo supericialmente. En el fondo son reservas de significado (...) Los museos son depósilos de clasificaciones, aunque no simples depositarios de reliquias estáticas. Ellos son entonces dinámicos, transtomadores en los que la energia es convertida, redistribuida y emitida:"

4. cuando coleccionamos objetos estamos coleccionando información. Lo que propongo es que el énfasis debe ser puesto en la información más que en el objeto. El objeto puede convertirse en un fetiche que de ser simplemente adorado, impide nuestro entendimiento del objeto en si y su lugar en la sociedad. $Y$ en el proceso de ser adorado puede a veces deshacerse en nuestras manos".

2 -Ultimada empresa materialista, los museos parecen reemplazar personas por objetos. Exirayendo de ellos vida y pensamientos, con la creencia que a través de ello, se inmortalizarán el espiritu de civilizaciones pasadas". 
tor, llámese el visitante al museo, lectura que dependerá a su vez del contexto cultural al que el/ella pertenezca y "la gramática" utilizada por el museo, el modo como éste estructura el mensaje y nos presenta el objeto.

\section{II.- La gastronomía como patrimonio intangible}

Aunque los museos siguen organizando sus actividades en torno al patrimonio tangible y mueble, hoy en día son más concientes de la intangibilidad tras sus objetos, llámese los valores en ellos, las cualidades que los hacen ser coleccionados y separados del sistema en el cual estaban inscritos ${ }^{5}$. Igualmente la valoración del patrimonio intangible fuera de los museos, pero con el que las colecciones de éstos guardan vínculos directos o indirectos van creciendo en importancia ${ }^{6}$.

Instituciones tales como la UNESCO han tomado los últimos años acciones para promover la protección y difusión del patrimonio inmaterial, entre estas acciones tenemos programas tales como "Obras maestras del patrimonio oral e inmaterial de la humanidad" (155 sesión, Octubre- Noviembre 1998, Consejo Ejecutivo de la UNESCO). En muchos paises desde los años 50 se han ido desarrollando sistemas de protección que consideran este tipo de herencia cultural. Sin embargo, con excepción de Francia (Arellano: 2002), ningún país ha desarrollado un sistema específico para el patrimonio gastronómico ${ }^{7}$, a pesar de que la UNESCO incluye a éste como parte del patrimonio intangible a proteger:

"Podria definirse el patrimonio intangible como el conjunto de formas de cultura tradicional y popular o folclórica, es decir,

\footnotetext{
5 De alli que también que cada vez cobra mayor importancia la preservación insitu o contextualizada del patrimonio tangible.

6 A este respecto es interesante considerar los apartados del programa del AFRICOM (Comité Internacional de Museos para África) dedicados a los recursos de la herencia cultural intangible, el rol que los museos deberian jugar en su salvaguardo, los medios e instrumentos recomendados.

7 México tiene un Programa de Gastronomia bajo la Dirección General de Culturas Populares, sin embargo este programa se centra más en la integración y recuperación de recetarios.
}

las obras colectivas que emanan de una cultura y se basan en la tradición. Estas tradiciones se transmiten oralmente $o$ mediante gestos y se modifican con el transcurso del tiempo a través de un proceso de recreación colectiva. Se incluyen en ellas las tradiciones orales, las costumbres, las lenguas, la música, los bailes, los rituales, las fiestas, la medicina tradicional y la farmacopea, las artes culinarias y todas las habilidades especiales relacionadas con los aspectos materiales de la cultura, tales como las herramientas y el hábitat". (UNESCO: 2001, negritas propias)

Es imprescindible tener en cuenta que en el caso del patrimonio gastronómico, como otros tipos de herencia cultural, los límites clasificatorios son más bien difusos y los nexos con otros formas de producción cultural son innumerables, diferentes formas de creatividad popular se sobreponen e interactúan en la producción y constante evolución del patrimonio. Muchas veces las actividades de investigación, preservación y difusión del museo se extienden más allá de la estructura física de esta institución; interesante es reconocer que el campo de su investigación comprende también los diferentes "espacios culturales" en los que su colección y la comunidad con la que trabaja se inscriben. Siendo un "espacio cultural" un concepto antropológico referido no sólo a espacios geográficos específicos, sino también a una serie de lugares (no sólo físicos) en los que una forma de tradición popular se repite y se modifica de manera constante. En el caso de la gastronomía los espacios culturales estarian conformados por las llamadas rutas gastronómicas, últimamente materia de investigación de especialistas en estos temas (Benavides, en prensa). En este caso tanto el espacio cultural como la tradición culinaria en sí serian considerados herencia cultural inmaterial de una sociedad. Resulta difícil imaginar que los museos avocados generalmente a la preservación material de los objetos puedan jugar un rol importante cuando esta actividad de salvaguardar el patrimonio gira en torno a algo inmaterial. Sin embargo los museos pueden participar en esta 
empresa, siendo dos las formas más comunes de preservación que pueden incluir: recopilar la información actual a través medios de registro visuales, escritos o sonoros; y facilitar la supervivencia de estas tradiciones ayudando a las personas involucradas y asistiendo en la transmisión de estos conocimientos a generaciones futuras. Quizás la segunda opción resulta siendo la mejor a largo plazo, pues permite en cierto modo que la actividad, vale decir la gastronomía, se mantenga y evolucione. Así, en el caso particular del patrimonio gastronómico, aunque podamos registrar los procedimientos, recetas, las actividades y producciones culturales relacionadas con su consumo, etc. El acto tal cual, real de creación no tiene una forma física, o al menos no una permanente, el acto de creación es inmaterial, y es difícil predecir la futura evolución de esta actividad.

Los museos a través de su respaldo institucional pueden identificar y dar reconocimiento oficial a aquellos que desarrollan estas prácticas culinarias tradicionales. Un ejemplo de ello es el Museo Nacional de Arqueología, Antropología e Historia (Lima, Perú) que realiza una vez al año un concurso de dulces y postres limeños. Muy importante es también promover actividades específicas dentro del departamento de investigación del museo, fomentando trabajo de gabinete y de campo; proyectos de registro y documentación con las comunidades pertinentes.

Interesante de recordar en el caso del patrimonio inmaterial es la concepción holística que las comunidades respectivas tienen de éste, sobre todo en el caso de muchas comunidades indígenas:

"there is something artificial in separating out traditional knowledge of medicine, of husbanding nature, of religion, and so on, since they are interdependent and part of a whole conception of life and natural cycles" (Prott, 2000).

\footnotetext{
8. ...hay algo de artificial en separar el conocimiento tradicional de medicina, de religión entre otros, en tanto ellos son interdependientes y parte de una concepción total de vida y ciclos naturales".
}

Así también la gastronomía más que una actividad tradicional aislada, es un proceso social, íntimamente relacionado con otras actividades tales como la pesca y caza, las tradiciones religiosas y los rituales, actividades de socialización propias de cada cultura, entre otros. Cristina Padilla nos lo recuerda al hablarnos de la importancia de la gastronomía en los hoteles para inmigrantes, uno de ellos, hoy museo del Immigrante en Buenos Aires:

\begin{abstract}
"A esto se denomina ahora patrimonio intangible: aquel que antes que materialidad o monumento es canción, sabor, aroma, recetas contadas, maneras de acomodar el cuerpo a ritmos especiales de amasar, hornear, freír; disposición de la mesa, formas de celebrar, maneras de recibir al otro invitado a la mesa. Diferenciarse para compartir, compartir para intercambiar, intercambiar para integrarse.
\end{abstract}

\section{(...) La incansable repetición de estas recetas y secretos culinarios es donde anida el espíritu de lo intangible." (Padilla: 2002, 56)}

En este sentido, cobran importancia propuestas como la de Prott, quien nos presenta un planteamiento con objetivos, necesidades y medios a usar para resguardar cada uno de los diferentes tipos de patrimonio inmaterial; en este listado podemos observar como medios similares pueden contribuir a la preservación de diferentes tipos de patrimonio inmaterial. Así objetivos señalados por este autor para la protección de otro tipo de patrimonio inmaterial pueden resultar igualmente válidos para el gastronómico, como la conversión de éste en una fuente de ingreso, objetivo que podría añadirse a los de mantener los hábitos culinarios distintivos, mantener estilos de vida sustentables y promover una dieta saludable. Como necesidades para alcanzar estos objetivos, se deben considerar no sólo la conservación del knowhow (o "saber cómo") de la actividad en sí, sino también la disponibilidad de ingredientes res- 
pectivos a través de actividades tradicionales tales como la pesca, caza y cultivos, así como los implementos de cocina tradicionales. Los museos pueden colaborar en la consecución de estos objetivos y la difusión de éstas necesidades a través de programas activos de investigación y comunicación, que vayan más allá del registro de recetas (que por ejemplo Prott menciona como un medio). Los museos pueden también participar en la adopción de un sistema de tesoros de culturas vivas bajo los parámetros señalados por la UNESCO, la difusión de la necesidad de protección y establecimiento de eco-reservas, y de niveles óptimos de calidad en el turismo cultural. Así, recordando que el patrimonio inmaterial, incluyendo la gastronomía es influenciado por tantos otros procesos sociales, incluyendo aquellos que pueden hacerlo más vulnerable a su resguardo, tales como globalización, turismo y comodificación; los museos están llamados a desarrollar a través de sus diferentes áreas actividades y exhibiciones que permitan la reflexión de la comunidad sobre estos temas.

\section{III.- Cambios dentro del museo: la visita como una experiencia integral}

Son muchos los cambios en los museos que facilitan la inclusión en estas instituciones de programas y actividades alrededor del patrimonio cultural intangible, y en este caso especifico la gastronomía. Quizás a partir de los años 70 se hace más patente sobre todo en los museos de Norte América, pero también en ciertas localidades de Latinoamérica, que las diferentes actividades de estas instituciones giran en torno no sólo a las colecciones sino también al público visitante y a la sociedad o comunidad a la cual el museo pertenece. Desde el punto de vista de la museología teórica se da también una mayor importancia al estudio de los visitantes. De este modo a partir de los años 80 , además del gran impulso que reciben las áreas educativas y de comunicación, los especialistas prestan mayor atención a la experiencia del público y al análisis de las visitas de éste como experiencias integrales, en las que diferentes factores juegan un rol importante no sólo en el aprendizaje, sino en el disfrute de la visita y la motivación para retornar al museo y participar en actividades organizadas por éste en ocasiones futuras.

Muy importante dentro del área educativa es la redirección que se está tomando en los museos en cuanto a la utilización de los objetos: de ser usados estos como "evidencia de" pasan a ser usados como elementos que provocan experiencias, la segunda tendencia que en realidad se fusiona con la primera valora el significado emotivo sobre el cognitivo. Como lo señala Hein, esta fusión identifica lo experimental con lo empático, y al recurrir a la experiencia de la realidad, da prioridad a la evocación del sentimiento. Según la autora,

"Experience is taken to be inherently emotional; indeed, the very idea of affectless experience is sometimes suggestive of a pathological cast of mind. The public demands that ideas be so presented as to make them imaginable... In holding that holistic engagement through multidimensional experience, rather than distanced contemplation, answers a common human need, these museums are taking a novel epistemological and ontological stand. The reality they aim to construct is cognitively and emotively totalizing" (Hein, 2000: 80).

¿Qué relación guardan esta nueva tendencia en los museos con el patrimonio gastronómico? Si consideramos, por un lado la dimensión intangible de los objetos que resguardamos, investigamos y difundimos en los museos; y por otro lado la naturaleza holística del patrimonio, por cierto no sólo tangible sino también intangible, muchos serán los vínculos

\footnotetext{
9 "La experiencia es considerada por ser inherentemente emocional; en verdad, la mera idea de una experiencia sin afecto es algunas veces sugerente de alguna patologia mental. El público demanda que las ideas sean presentadas de un modo que puedan ser imaginables... Manteniendo este compromiso holistico a traves de la experiencia multidimensional por sobre una contemplación distanciada, responde a una necesidad humana, estos museos toman una novedosa posición epistemologica y ontologica. La realidad que ellos intentan a construir es cognitiva y emocionalmente totalizante*.
} 
que hallaremos entre gran parte de las colecciones en los museos y el tema gastronómico.

"Museums still collect objects and still take pride in the size and quality of their collections. They continue to preserve and study objects, but their chief occupation is neither to discover nor to keep them. It is to foster the intersubjective constructions that object elicits. No longer content to be styled as graveyards or department stores, museums now are cast as impresarios of meaning performances. They have become manufacturers of experience." (Hein, 2000: 55) ${ }^{10}$.

Es tarea de los museos hacer de esos vínculos la oportunidad de crear experiencias más cercanas a la cotidianeidad de los visitantes... ¿y qué más cotidiano que la ingesta de alimentos?. Interesantes exhibiciones y actividades pueden ser organizadas en torno a temas gastronómicos u otros relacionados a ellos, desde un mayor conocimiento de recursos vegetales en determinada región, hasta los rituales y costumbres que acompañaron o acompañan la ingesta de alimentos en determinadas culturas. Aunque de primera impresión serían los museos con colecciones de historia social, antropología y arqueología aquellos en los que la gastronomía podría jugar un papel importante; museos de arte, de personajes en particular", filmotecas y similares también pueden utilizar este tema tan cercano a todos. La representación de la comida y de temas relacionados a la gastronomía en la literatura, cine y otras

\footnotetext{
10 "Los museos aún coleccionan objetos y aún se sienten orgullosos del tamaño y calidad de sus colecciones. Continúan preservando y estudiando objetos, pero su ocupación principal no es ni descubrirlos ni conservarlos; es fomentar las construcciones inter subjetivas que el objeto tiene. No más satisfechos con ser cementerios o tiendas de deparlamento, los museos son ahora forma. dos como intérpretes de significados. Se han convertioo en creadores de experiencia."

11 Existen muchos personajes importantes tales como Neruda o Goethe, que hicieron interesantes apreciaciones sobre la gastronomia, $\theta$ incluso como en el caso del primero tomaron el tema culinario como tema parte de su trabajo. (basta leer algunas de las Odas elementales). En el caso del segundo, muchos pasajes de sus libros, notas de viaje, cartas, etc. reftejan la importancia que el poeta le daba a la buena comida y bebida, además de ser un testimonio de la gastronomia de la época. Al respecto, novedosas publicaciones aparecieron el año del 250 aniversario del autor, tales como Nagel, Joachim (1999) Zu Gast be Goethe -De huésped con Goethe-, Brunner Peler (1995) Zu Tisch mit Goeth - A la mesa con Goethe- o Schulz, Joachim Essen und Trinken mit Goethe (1999) - Comiendo y bebiendo con Goethe.
}

artes no es una excepción sino una presencia constante en diferentes regiones y tiempos, finalmente las expresiones artísticas, son también resultantes de procesos sociales al igual que la tradición gastronómica. De este modo, el tema de la gastronomía y aquellos relacionados con ésta pueden ser interesantes vínculos para los espectadores entre no sólo pasado y presente, sino también diferentes regiones y expresiones culturales.

Además de las exhibiciones los museos también pueden integrar en sus programas, material educativo, boletines y publicaciones diversas no sólo sobre el patrimonio gastronómico en sí, sino también con temas que se vinculan y sobreponen a éste. Otras actividades importantes para la difusión de este patrimonio lo constituyen los festivales gastronómicos, concursos, juegos para niños, talleres, excursiones, etc. Estos últimos medios de difundir el interés por la gastronomía suelen ser además medios muy efectivos de aprendizaje:

"Las presentaciones que conllevan información importante para las experiencias comunes de la audiencia ganarán mayor atención (todas aquellas que tengan que ver con la vida cotidiana, por ejemplo). La información que tiene relevancia personal es codificada con mayor profundidad que otras $y$, por tanto, se recordará con mayor facilidad." (Morales Miranda, 2001: 110)

La experiencia del museo, no sólo a de ser una experiencia visual y personal, sino también una experiencia que definitivamente involucra todos nuestros sentidos y que es influenciada también por factores sociales: si las actividades dentro de los museos son realizadas en grupos y van más allá del papel de espectador, es más probable que las impresiones adquiridas sean más duraderas, originen un mayor interés por el tema y futuros nuevos contactos entre el visitante, el museo e instituciones similares ${ }^{12}$.

\footnotetext{
12 Según Falk and Dierking (1992), son tres los contextos que se sobreponen $\theta$ interactúan moldeando la experiencia resultante de la visita aun museo: el contexto personal, el contexto social y el contexto fisico. Cada uno de ellos con una serie de variables, factores determinantes de una experiencia grata.
} 
Aún más allá del área educativa, la gastronomía puede constituir parte relevante de la experiencia del museo como una experiencia integral, en la que no sólo las exhibiciones y las actividades directamente relacionadas con éstas son elementos importantes, sino también los otros servicios que el museo ofrece como suplementarios, como por ejemplo la tienda y la cafetería. Estos servicios, aunque no forman parte de los servicios centrales del museo, forman parte de la visita como una experiencia integral. Como lo menciona Kotler (2000), para muchos los museos constituyen una opción más dentro de la amplia oferta de tiempo libre, tiempo libre que es consumido no sólo en forma individual sino en grupos, ya sean de amigos, familias, escolares, etc. Para estos grupos ciertos espacios del museo constituyen espacios de socialización, como por ejemplo las cafeterías y restaurantes de los museos. Estos espacios no tienen porque ser unidades apartes de las salas de exhibición y de las colecciones del museo; todo lo contrario pueden reforzar y hacer más cercana la experiencia de la visita y también seguir los lineamientos de la misión y los objetivos del museo. Un interesante ejemplo de lo mencionado es el Restaurant "El Ekeko" del Tropenmuseum (Museo de los Trópicos) de Ámsterdam, que ofrece a sus visitantes sólo platos y snacks que constituyen expresiones gastronómicas de las culturas presentes en sus colecciones; $y$ de las diversas exhibiciones, conferencias, actividades, etc. relacionadas con éstas.

\section{IV.- La gastronomía como tópico integrador entre sociedad y museo:}

Aún más interesante para la comunidad es la participación activa de sus miembros en proyectos llevados a cabo en los museos. En el tema gastronómico, como en otras tradiciones populares, algunos miembros de la comunidad son "expertos" del tema, vale decir portadores de los conocimientos que hacen posible la continuidad y evolución de las tradiciones culinarias. Debido a ello, es trascendental como política del museo crear lazos con la sociedad, no sólo considerando a éstos como visitantes y espectadores, sino como conocedores y creadores, en trabajo conjunto con los profesionales del museo. La gastronomía podría constituir en un buen mecanismo para la creación de contactos más cercanos entre las comunidades y los museos: "assimilating neighborhoods to museums and museums to neighborhoods" (Hein, 2000: 51) ${ }^{13}$.

Es así, que a través de los museos se puede empoderar también a aquellas personas que son las portadoras y transmisoras de esta actividad tradicional importante. Quizás aún más importante de recordar es que la gastronomía tal como otras expresiones de la producción cultural tradicional sea con propósitos utilitarios o artísticos, representa una forma muy valiosa de expresión cultural, un capital de confianza en sí mismo que es especialmente importante en los países en vías de desarrollo (Proot, 2000). Los museos pueden ampliar aún más el campo de acciones con referencia al tema del patrimonio gastronómico, a través de la formación de redes tanto nacionales como internacionales que ayuden a garantizar que las partes interesadas, generalmente comunidades culturales, puedan también disfrutar de los derechos económicos, de autoría, y otros resultantes de la investigación, creación y difusión del patrimonio en cuestión.

El patrimonio culinario es a su vez un elemento básico integrador de culturas; hecho que puede ser utilizado por aquellos museos inscritos en ciudades grandes con poblaciones multiculturales, como es el caso de un buen número de ciudades latinoamericanas. A través de diferentes actividades se puede promover el respeto y valoración de las diferentes expresiones culturales que convergen en un espacio geográfico. El confrontar un aspecto tan presente y cotidiano en todas las culturas permite no sólo un mayor autoconocimiento y revaloración de la identidad propia, sino también una menor desconfianza del "otro", un mayor respeto por la alteridad, una convivencia alejada de prejuicios. Recordemos que todas las cul-

\footnotetext{
13. Asimilando los vecindarios a los museos y los museos a los vecindarios".
} 
turas se definen a sí mismas a través de su relación con otras, especialmente las instituciones y expresiones culturales que las representan. En el caso de la alimentación y la tradición gastronómica, como en otras de estas expresiones, son muchos los ejemplos de mestizaje, no sólo por la utilización de alimentos provenientes de diversas regiones, sino también por influencias y adopciones de modos de producción, recreación y consumo de éstos. Vivimos pues, sobretodo en las grandes ciudades, en comunidades fronterizas, en las que los hábitos y costumbres incluidas los gastronómicos son tomados prestados de otros, son intercambiados sin ser muchas veces sus miembros concientes de los elementos tomados del "otro". Una mayor conciencia del proceso y resultado de estos intercambios constituye definitivamente una mayor conciencia de lo que nosotros mismos somos y a donde vamos. Es quizás también trascendental recordar que es en las ciudades donde a la vez se hace más tangible el rápido reemplazo de las culturas locales inmateriales por una cultura estandarizada internacional, promovida como lo señala la UNESCO, no sólo por la "modernización" socioeconómica, sino también por el enorme progreso de las técnicas de información y transporte.

\section{V.- Turismo, gastronomía y museos}

Los museos y la gastronomía forman parte de casi toda experiencia turística o al menos de la oferta hecha a los turistas. Al igual que la visita a un museo, el alejarse por un tiempo del lugar habitual de trabajo, estudio o vivien$\mathrm{da}$, constituye una experiencia integral en la que todos nuestros sentidos entran en juego, muchas veces de un modo más atento que en el de la vida diaria en nuestro medio habitual. Como lo señala Castro (1998) cuando éste menciona el caso del autodenominado Museo del Jamón en la "milla de oro" madrileña ${ }^{14}$, este establecimiento (dedicado sobretodo a la venta de jamón serrano y similares), al igual que

14 Area de la ciudad de Madrid con mayor densidad de museos, incluyendo entre otros al Museo del Prado. algunos museos del vino, gastrotecas y vinotecas, reflejan no sólo "que la buena bebida y la buena comida pueden llegar a producir una fruición equiparable a la que experimentamos al visitar un museo o una muestra artística" (Castro: 1999, 129), sino también que se viene dando una transformación del concepto del museo, como algo más próximo al ciudadano. Así, muchos lugares o instrumentos creados a partir del siglo XIX para observar como parte de la experiencia de viajar y conocer están ligados a involucrar todos nuestros sentidos en actividades que integran modos de socializar, observar y relacionarse con lo propio y lo ajeno a través de la comida y la bebida; entre estos espacios se encuentran por ejemplo los cafés: "The street level was lined with many small businesses, shops and especially cafés. The last of these have come to be known all over the world as signs of la vie Parisienne, particularly as generations of painters, writers and photographers have represented the patterns of life in and around them, beginning with the Impressionists in the 1860s" (Urry: 2002, 125) ${ }^{15}$. Esta idea está estrechamente relacionada a su vez con aquello que da forma a la identidad de una región, localidad o ciudad; todas las expresiones culturales, y no solo la arquitectura o el arte, forman parte de esta identidad: Thus territory is less central to national self-definition' and more important are specific places, landscapes and symbols including various forms of consumption including food, drink and art" (Maier and Lowenthal quoted by Urry: 2002, 158 $)^{16}$. Importante es entender que estas formas de consumo, mencionadas por Urry, no se dan de forma aislada, sino integrándose y sobreponiéndose, del mismo modo que nuestras experiencias no aíslan lo percibido por cada sentido, sino que cada percepción es ingrediente de una experiencia total.

\footnotetext{
15 -El nivel de la calle está alineado con muchos comercios pequeños, tiendas y especialmente cafés. Estos últimos se han convertido en el mundo entero en simbolos de la vida parisina, particularmente en tanto generaciones de pintores, escritores y fotógrafos han representado los estilos de vida en y alrededor de ellos, comenzando por los impresionistas en los $1860^{\circ}$

16 "Entonces el territorio es menos importante para la autodefinición nacional; siendo más importantes lugares especificos, paisajes y simbolos incluyendo varias formas de consumo como la comida, la bebida y el arte".
} 
Una interesante propuesta en la que se combinan arte y gastronomía en México, es aquella planteada por Bernard y Domínguez, a través de la creación de rutas en Ciudad de México, Puebla y Oaxaca, ciudades declaradas Patrimonio de la Humanidad por la UNESCO, sobre cuya herencia cultural tangible y monumental se han realizado muchas actividades de investigación, protección y difusión, pero cuya herencia culinaria, recién empieza a ser revalorada $^{17}$. A este respecto las autoras llaman la atención sobre la carencia de museos relacionados con el tema culinario y la escasez de material bibliográfico con un enfoque históricoantropológico. De allí la importancia de no sólo combinar estaciones diversas (muchas de estas rutas incluyen la combinación de museos, palacios, iglesias y mercados), sino de relacionar contenidos y actividades entre los diversas instituciones participantes.

Los museos e instituciones similares tienen diversas posibilidades para formar parte del circuito turístico y a la vez promover la preservación y difusión del patrimonio gastronómico, contribuyendo además al desarrollo sustentable de las comunidades con las que están involucrados. Una de las posibilidades es la de constituirse en mediadores entre los portadores y creadores del patrimonio gastronómico y las agencias organizadoras de los paquetes turísticos, los turistas mismos o los visitantes en general. De este modo Marc Patry nos presenta tres casos distintos en las Américas (Canadá, México y Chile), todos ellos sin embargo parte de la red Model Forest en los que diferentes entidades sirven de mediadores y coordinadores entre los creadores del patrimonio cultural viviente y la industria turística ${ }^{18}$. Como Patry bien lo menciona este papel coordinador

\footnotetext{
17 A nivel latinoamericano el tema del patrimonio gastronómico y el turismo cultural, es ya motivo de un congreso anual: Patrimonio gastronómico y turismo cultural en América Latina y el Caribe, llevado a cabo en Puebla, México a partir de 1998, como iniciativa de la UNESCO y que $s \theta$ repite anualmente. Existen también ya rutas gastronómicas estructuradas en Argentina y Brasil. En Argentina las rutas propuestas son: la ruta del caprino, del marisco, de la trucha, de la pera y manzana, de la fruta fina, del ovino y la ruta del ciervo (Schlueter, 2002)

${ }^{18}$ En el caso de Canadá el proyecto incluye a productores de jarabe de maple en Ontario, en México la mediadora es una ONG e incluye a comunidades indigenas de Kalakmul; y en Chiloé, Chile, la fundación "Con todos" a permitido organizar mejor a los pobladores para llevar a cabo programas de agroturismo (en los que por cierto la gastronomía de la zona juega un papel importante).
}

podría ser llevada a cabo también por los museos brindando así a sus visitantes aún una experiencia más cercana al patrimonio cultural viviente. De hecho un museo que realiza este tipo de papel de intermediario es el museo de culturas populares de la Universidad de Heredia de Costa Rica, quienes desarrollaron hace algunos años el concepto del museo productivo, mediante el cual trabajan en conjunto con artesanos populares de la localidad.

Otro caso interesante de trabajo en redes, combinando turismo cultural y patrimonio gastronómico lo es la Red de Herencia Culinaria Europea (European Culinary Heritage Network), que tiene como base el mayor acercamiento de los consumidores a través de Europa a los sabores regionales; el desarrollo de pequeños negocios y la preservación de las características particulares de cada área. Como ventajas la formación de redes y el trabajo en conjunto ofrecen a sus miembros la inserción en un sistema de relaciones superior; así las redes regionales se pueden unir a las nacionales y a las que agrupan países como es el caso de esta red de alcance europeo. Las redes permiten el acceso a grandes volúmenes de información para todas las instituciones participantes; así mismo pueden utilizar de un modo más eficiente los recursos para promocionarse y posicionar una imagen en conjunto.

Ejemplo también interesante del trabajo en redes incluyendo museos (aunque no sólo de índole culinaria) es el de los economuseos en Québec. Estos nacen bajo la concepción de hacer auto financiables museos (en el sentido más amplio) y conservar no precisamente objetos, sino tradiciones productivas locales. Así, se combinan conceptos de economía y museología, obteniendo beneficios no sólo cuantitativos, llámese ingresos propios y autofinanciamiento; sino también cualitativos, la preservación de técnicas de producción tradicionales. Los economuseos se presentan como pequeños negocios en los que los productos ofrecidos han sido elaborados a través de técnicas tradicionales locales, los visitantes no sólo tienen la 
posibilidad de adquirir estos productos, sino también de conocer de un modo más cercano los procedimientos de producción, a través de la participación en los talleres de producción, un servicio de interpretación del patrimonio y la posibilidad de acceder a documentación y mayor información sobre el tema del economuseo. El valor añadido de estos economuseos lo constituye también un marco geográfico que refuerza el contenido cultural del patrimonio intangible en cuestión y a la vez favorable al turismo. Esta red de economuseos forma parte de un circuito turístico y hoy en día abarcan más de 36 estaciones, de las cuales 9 están ligadas a técnicas de producción de productos alimentarios tradicionales: el museo de la harina, del queso, de la miel (que incluye su aplicación en chocolatería y pastelería tradicional), vino, jarabe de maple ( $y$ bebidas alcohólicas derivadas), arenques ahumados, ostras, whisky y cerezas silvestres.

Tanto los economuseos de Canadá, como muchos ecomuseos en áreas rurales, significan un enlace de 2 temas muy importantes dentro de la agenda de los museos con relación a su comunidad: Por un lado la conservación de herencia cultural intangible a través de la preservación de técnicas culinarias (y similares); $y$ por otro lado el desarrollo económico sostenible de la comunidad a la que el museo sirve. El desarrollo económico, sobre todo en zonas rurales previene la migración de su población, especialmente los más jóvenes, hacia las ciudades; promoviendo a su vez la conservación de estilos de vida y otras formas de tradiciones locales particulares.

Significativos son también en el caso no sólo de las áreas rurales, sino también en las ciudades los diferentes museos comunitarios y los museos de cultura popular. En estos últimos, el tema gastronómico juega un papel importante, siendo parte de uno de los temas centrales de las exhibiciones y actividades con la comunidad. Como ejemplo tenemos: El Museo de Cultura Popular de la Universidad de Heredia en Costa Rica y el Museo Nacional de Culturas
Populares de México. El primero realiza actividades y talleres con niños, además de tener un programa completo de rescate, reactivación y difusión de tradiciones populares, entre las que la gastronomía juega un rol importante. EI segundo incluye dentro de su programa de exhibiciones temporales, algunas de temática gastronómica: "La cultura del maíz", "la cultura del café", "Muéganos, pelucas y rosquetes" (en torno a la panadería regional), "una pizca de sal", "dime qué comes...", "Las cocinas de México", entre otras. El Museo Nacional de Cultura Popular (Lima, Perú), organiza a su vez actividades relacionadas con las tradiciones culinarias diversas como el concurso de tantaguaguas $^{19}$, el Museo Nacional de Antropología, Arqueología e Historia del Perú también realiza anualmente el concurso de postres y dulces tradicionales, con gran acogida de un público que quizás no forma parte regularmente de los visitantes a museos. Es interesante en el caso mexicano que el museo se encuentra inscrito dentro de la Dirección General de las Culturas Populares, que considera dentro de sus programas (la mayoría de ellos con énfasis en el patrimonio intangible) uno de gastronomía que tiene como objetivo el llevar a cabo investigaciones de campo y gabinete para la integración o recuperación de recetarios. Los centros culturales y museos comunitarios integrados por esta Dirección, son como estaciones con actividades propias de acuerdo al calendario de festividades de cada región, pero a su vez con actividades comunes a todos de acuerdo al programa nacional de gastronomía.

Existen también en Latinoamérica algunos museos cuya razón de ser es precisamente algún producto alimenticio de vital importancia en la gastronomía de una región o país y que por lo mismo también juega un papel relevante en la economía local o regional. Interesante en el caso latinoamericano es comprender que la mayoría de estos museos resaltan la estrecha relación entre desarrollo

\footnotetext{
19 Panes tradicionales de ciertas zonas de la sierra del Perú en forma de muñe$\cos 0$ animales, preparados especialmente en noviembre como parte de las celebraciones del dia de los muertos.
} 
sustentable y herencia cultural, destacando no sólo la preservación y respeto por el medio ambiente, sino también por las técnicas tradicionales no agresivas a la naturaleza. Un buen ejemplo de ello es el Museo del café en Chiapas, México.

Europa presenta aún un mayor número de museos que tienen como tema principal productos alimenticios tradicionales de gran significación cultural regional en ese continente, destacando quizás entre estos productos el queso (en Holanda, España y Francia), el vino (en España, Italia, Alemania y Francia), el aceite (en Italia) y la cerveza (Bélgica, Holanda, España, Alemania). Sin embargo a diferencia de museos de temática similar en Latinoamérica, en Europa muchos de estos museos pertenecen generalmente a negocios familiares de larga tradición en las regiones, siendo por lo tanto de naturaleza privada; en tanto que en Latinoamérica, los pocos que existen tienen sus orígenes en la organización de comunidades campesinas, nacen bajo esta iniciativa, o con el auspicio del gobierno nacional o local.

\section{VI.- A modo de conclusión}

Son diferentes los temas parte de la cultura de cada región, localidad, etc. que de algún modo forma parte del tema patrimonio gastronómico y de sus muchos puntos de partida y llegada como lo menciona Gabriela Benavides (en prensa). A lo largo de este camino con sus ramificaciones y paradas, son muchas las intersecciones en las que los museos pueden acercarse al tema del patrimonio gastronómico y de este modo acercarnos a nuestra propia identidad, a un mayor conocimiento del otro en el tiempo y en el espacio, a la revalorización de un capital intangible como lo es el amor propio, elemento indispensable para cualquier proyecto o iniciativa de desarrollo sostenible. Quizás convendría recordar la respuesta que Douret nos da sobre "lo nuevo" en la nueva museología:

"Lo que es nuevo, es el reconocimiento por parte de los museólogos, mediadores culturales y segmentos interesados del público, que los museos son, o pueden ser, activos colaboradores de actores locales y regionales, en el proceso de aprender haciendo, posibilitando competencias en acción, empoderando a la colectividad en el uso de la exhibición como medio o mediación comunitaria, hacia el salvaguardo del patrimonio como un bien común en una sociedad civil". (Douret: 1999, 8)

Rhina I. Colunge Rosales Instituto de Investigación Escuela Profesional de Turismo y Hotelería Universidad de San Martin de Porres rhinushka@yahoo.com 


\section{Bibliografía}

\section{AFRICOM}

1999 Final Endorsed Programme. Constituent Assembly, 3-9 October 1999, Lusaka, Zambia. www.africom.org

Arellano, Alejandro

2002 "Echando una mirada al patrimonio", Turismo y patrimonio, 2000, Nro 2, Lima: Universidad de San Martín de Porres

\section{Bernard y Domínguez}

2002 "Gastronomía. La herencia olvidada", Schlueter \& Norrild (2002), Turismo y patrimonio en el siglo XXI. Buenos Aires: Centro de Investigaciones y Estudios Turísticos.

Benavides Gabriela, compiladora

en prensa. "Presentación", Alimentación y tradición gastronómica. Discusiones en torno a la construcción del patrimonio gastronómico. (Colección Patrimonios - Gastronomía 2), USMP, Lima.

\section{Cabrera, Adriana}

1999 The Qualitative Profitability and Productivity of Economuseums.

Amsterdam: Reinwardt Academie.

Castro Morales, Federico

1998 Patrimonio, museos y turismo cultural: claves para la gestión de un nuevo concepto de ocio. Córdova: Servicio de publicaciones de la Universidad de Córdova.

De Carli, Georgina

1997 The Productive Museum. Proposal for change. Instituto Latinoamericano de Museología, Universidad de Heredia, Costa Rica.

\section{Douret Paul}

1999 "Introduction": What's New in New Museology? Ecomuseology and Social Museology. International Bibliography. Ottawa: MINOM.
Falk \& Dierking

1992 The museum experience. Washington DC: Whalesback Books.

Hein, Hilde

2000 The museum in transition. A philosophical perspective. Washington DC: Smithsonian Institution.

ILAM, El museo del café, usuarios.lycos .es/cafemuseocafe

Kotler, Neil

2000 "La experiencia museística: cómo disfrutar todo lo que ofrece el museo", Revista de Museología. Nro 18, noviembre 2000. Madrid: Asociación de Museólogos de España.

Luxen, Jean-Louis,

The intangible dimension of monuments and sites. ICOMOS paper: www.international.icomos.org/luxen_eng.htm

Museo Nacional de Culturas Populares, www. cnca.gob.mx

Ohlson, Thord

2000 Regional culinary heritage. A European network for regional foods. Monografía presentada en la conferencia internacional "Local Food \& Tourism", Chipre, 9-11 de noviembre del 2000.

Padilla, Cristina

2002 "Los sabores del patrimonio". Schlueter \& Norrild (2002), Turismo y patrimonio en el siglo XXI. Buenos Aires: Centro de Investigaciones y Estudios Turísticos.

Patry, Marc.

Museums: A link between living heritage and thetourism industry. www. chin. gc.ca/resources/ icom/english/collection/e_texte.mp

Prott, Lyndel,

Some considerations on the protection of the intangible heritage: claims and remedies, 
International Standards Section. Division of Cultural Heritage, UNESCO. www. folklife.si. edu/unesco/prott.htm

Schlueter, Regina

2002 "Turismo y patrimonio cultural. La gastronomía de la región pampeana". Schlueter \& Norrild (2002), Turismo y patrimonio en el siglo XXI. Buenos Aires: Centro de Investigaciones y Estudios Turísticos.

\section{Tellier Maryse}

1998 The transmission of savoir-faire in economuseums, Fondation International des economusees, Québec, Canada. www.economusees.com
UNESCO,

1989 Recommendation on the safeguarding of traditional culture and folklore adopted by the General Conference at its fifth session. Paris, 15 November 1989.

\section{UNESCO}

2001 Patrimonio intangible.

www.unesco.org/culture/heritage/intangi$\mathrm{ble} / \mathrm{html}$

Urry, John

2002 The Tourist Gaze. London: Sage Publications.

Washburn, W.

1984 "Collecting information, not objects", Museum news 62, 1984 (3) pp 5-15 